OPEN ACCESS

Edited by:

Peter Zilla,

University of Cape Town, South Africa

Reviewed by:

Fabrizio Buffolo,

University of Turin, Italy

Tanja Katharina Rudolph, Heart and Diabetes Center North

Rhine-Westphalia, Germany

*Correspondence: Birgid Gonska

birgid.gonska@uniklinik-ulm.de orcid.org/0000-0002-1015-5853

Specialty section: This article was submitted to Heart Valve Disease,

a section of the journal

Frontiers in Cardiovascular Medicine

Received: 09 July 2021 Accepted: 10 November 2021 Published: 08 December 2021

Citation:

Gonska B, Reuter C, Mörike J, Rottbauer W and Buckert D (2021) Vascular Access Site Complications Do Not Correlate With Large Sheath Diameter in TAVI Procedures With New Generation Devices. Front. Cardiovasc. Med. 8:738854 doi: 10.3389/fcvm.2021.738854

\section{Vascular Access Site Complications Do Not Correlate With Large Sheath Diameter in TAVI Procedures With New Generation Devices}

\author{
Birgid Gonska*, Christopher Reuter, Johannes Mörike, Wolfgang Rottbauer and \\ Dominik Buckert
}

Department of Internal Medicine II-Cardiology, University of UIm, UIm, Germany

Background: Vascular complications after transfemoral transcatheter aortic valve implantation (TAVI) are associated with morbidity and mortality. However, consistent predictors have not been identified yet. The size of the implantation sheath seems to play a role, though especially with new generation TAVI devices and their improved sheaths and delivery systems this remains uncertain.

Objectives: This study aimed to determine the incidence and predictors of access site-related vascular complications (VC) in the era of new generation TAVI devices.

Methods and Results: Four hundred consecutive patients receiving TAVI in an experienced tertiary care center were analyzed. VC occurred in 89 patients (22.25\%) with the majority being minor VC (21\%) and only $1.25 \%$ major VC. Possible predictors for VC were tested, and only peri-interventional dual antiplatelet therapy (DAPT) showed to be predictive for VC [OR 2.11 (95\% Cl 1.10-4.06, $p=0.025)]$. The female gender [OR 0.75 (95\% Cl 0.44-1.3), $p=0.31$ ], sheath to femoral artery ratio $>1.05$ [OR 1.18 (95\% Cl 0.66-2.08, $p=0.58)$ ], calcification of the access site vessel [OR $0.83(95 \% \mathrm{Cl}$ $0.48-1.42, p=0.48)$ ], known peripheral artery disease [OR 0.95 (95\% Cl 0.4-2.25, $p=$ $0.9)$ ], and $\mathrm{BMI} \geq 25 \mathrm{~kg} / \mathrm{m}^{2}$ [OR 0.69 (95\% Cl 0.41-1.19, $\left.p=0-19\right)$ ] were not predictive of VC. The larger sheath with 20 French even showed less VC than the smaller sheath with 16 French [OR $0.43(95 \% \mathrm{Cl} 0.25-0.74, p=0.002)]$.

Conclusions: Overall, the rate of major and minor VC was low in this study population (for major VC: rate of 1.25\%). Predefined risk factors were not associated with the occurrence of VC, except for peri-interventional treatment with DAPT. Especially, larger sheath size could not be identified as a predictor for $\mathrm{VC}$ in the setting of TAVI procedures performed with contemporary devices.

Keywords: transcatheter aortic valve implantation, vascular complications, bleeding, sheath size, calcification 


\section{INTRODUCTION}

Since the first transcatheter aortic valve implantation (TAVI) in 2002 by Cribier et al. (1), it has become the standard of care for inoperable patients or patients at high risk for surgical valve implantation (2). It has grown into a rapidly evolving alternative for patients at intermediate risk and is even progressing toward low-risk patients $(3,4)$.

The preferred approach for TAVI is transfemoral access, which has been associated with a better outcome than non-transfemoral access (5). With the first-generation TAVI devices, major vascular complications (VC) occurred in $\sim 10 \%$ of patients $(6-10)$. The occurrence of major VC proved to be associated with higher rates of morbidity and mortality $(6,11-13)$.

The growing experience with the management of percutaneous vascular access, development of new percutaneous suture devices, and improvements of the TAVI devices including implantation sheaths and delivery catheters have already led to a decline of VC compared with the beginning of TAVI. Nonetheless, VC is still one of the more common complications after transfemoral TAVI with incidences of 2 to $10 \%$ for major $\mathrm{VC}$, and a wide range of 2 to $29 \%$ for minor VC $(12,14-16)$.

In previous studies, a variety of risk factors have been identified to influence the occurrence of VC after transfemoral TAVI, such as female gender, obesity, peripheral artery disease (PAD), femoral artery diameter, sheath size or sheath to (ilio-)femoral artery ratio (SIFAR; SFAR, respectively), and calcification of the access site vessel and center experience $(7,13$, 17-20).

This study aimed to analyze the incidence of access siterelated VC in an all-comers patient cohort treated with the newest generation TAVI devices and to evaluate whether risk factors can be identified that have an impact on the occurrence of VC. Therefore, we assessed access site-related VC as defined by the updated standardized endpoint definitions for TAVI according to the second Valve Academic Research Consortium- (VARC2) criteria in patients treated with the newest generation TAVI devices (21). We chose to analyze TAVI devices with nonexpandable sheaths to ensure consistent sheath diameters: the Boston Scientific Lotus Edge (BLE) valve (20 French) and the Medtronic Evolut Pro/R (MEV) valve with a low-profile delivery system (EnveoPro, 16 French).

\section{METHODS}

In this retrospective single-center study, 611 consecutive patients treated with transfemoral TAVI for aortic valve disease between January 2019 and May 2020 were screened for treatment with either the self-expandable MEV Pro (size 23/26 or $29 \mathrm{~mm}$ ) and MEV R (size $34 \mathrm{~mm}$ ) or with the mechanically expandable BLE.

The decision for transfemoral TAVI was made by the interdisciplinary heart team according to the 2017 European Society of Cardiology/ European Association for CardioThoracic Surgery Guidelines for the management of valvular heart disease (22). The study was approved by the local ethics committee. All patients gave written informed consent.
All patients underwent preprocedural 256 multislice contrastenhanced CT, which was evaluated with a dedicated software (3mensio Structural Heart 9.1 software, Pie Medical Imaging B.V., Maastricht, The Netherlands). Besides the decision for the valve size, there was also an evaluation of the vascular access. The slice thickness for the evaluation of vascular access was 0.7 to $1.0 \mathrm{~mm}$. Routinely the access site was determined preprocedural and the size of the common femoral artery (CFA) was measured at the expected puncture position. Furthermore, calcification was semi-quantitatively classified in none, mild (calcification of not more than $25 \%$ of the circumference and not relevant protrusion into the lumen), or severe (calcification of more than $25 \%$ of the circumference, more than two spots, or relevant protrusion into the lumen) (Figure 1).

According to the hospital protocol, antiplatelet therapy was continued peri-interventionally whereas oral anticoagulation was stopped pre-procedurally. In these patients, single antiplatelet therapy was used peri-interventionally.

The decision for BLE or MEV device was made according to the experienced interventional cardiologist. Formally the BLE introducer set requires access vessel diameters of $6.5 \mathrm{~mm}$ or larger, the EnveoPro delivery system $5.5 \mathrm{~mm}$ or larger.

Transcatheter aortic valve implantation (TAVI) was performed in a hybrid catheterization laboratory under conscious sedation by an experienced operator team of four interventionalists with a standardized procedure protocol. First, the puncture of the non-access site CFA was performed under fluoroscopic control, and then the access site was cannulated under angiographic visualization via cross-over angiography from the non-access site, attempting for the puncture height predefined by the CT measurements. There was no use of ultrasound for the puncture. After the insertion of a 6 French sheath, angiography in an ipsilateral oblique view was performed to control the exact position of the puncture. Afterward, the vascular closure device was applied. For that matter, two Perclose ProGlide devices (Abbott Vascular, Santa Clara, California) were used at a 2-h angle (eleven o'clock and one o'clock). Then, the TAVI sheath was inserted over a stiff wire and heparin was administered to achieve an activated clotting time of 250-300 s. For the MEV Pro 23-, 26-, and 29-mm valve as well as for the MEV R $34 \mathrm{~mm}$ valve a 16 French Sheath (Cook Check-Flo Performer Introducer; Cook Medical, Limerick, Ireland) was used and later exchanged for the 16 French equivalent EnveoPro delivery system with an inline sheath, loaded with the valve. For the BLE the 20 French Boston Lotus Introducer sheath was inserted which remained in place throughout the procedure.

Before removal of the access site sheath, crossover access was obtained from the non-access femoral site with a 6 French pigtail catheter placed in the external iliac artery of the access site. Then, the access site sheath was removed and the ProGlides knots were pushed down and locked. Afterward, an angiography of the access site was performed. If necessary, dependent on the result of the angiogram and the discretion of the operator, either endovascular therapy (covered stent, percutaneous transluminal angioplasty) or manual compression was used to control the access site in case of a leak. Closure of the non-access site was achieved by 6 French Angio-Seal devices (Terumo Europe 

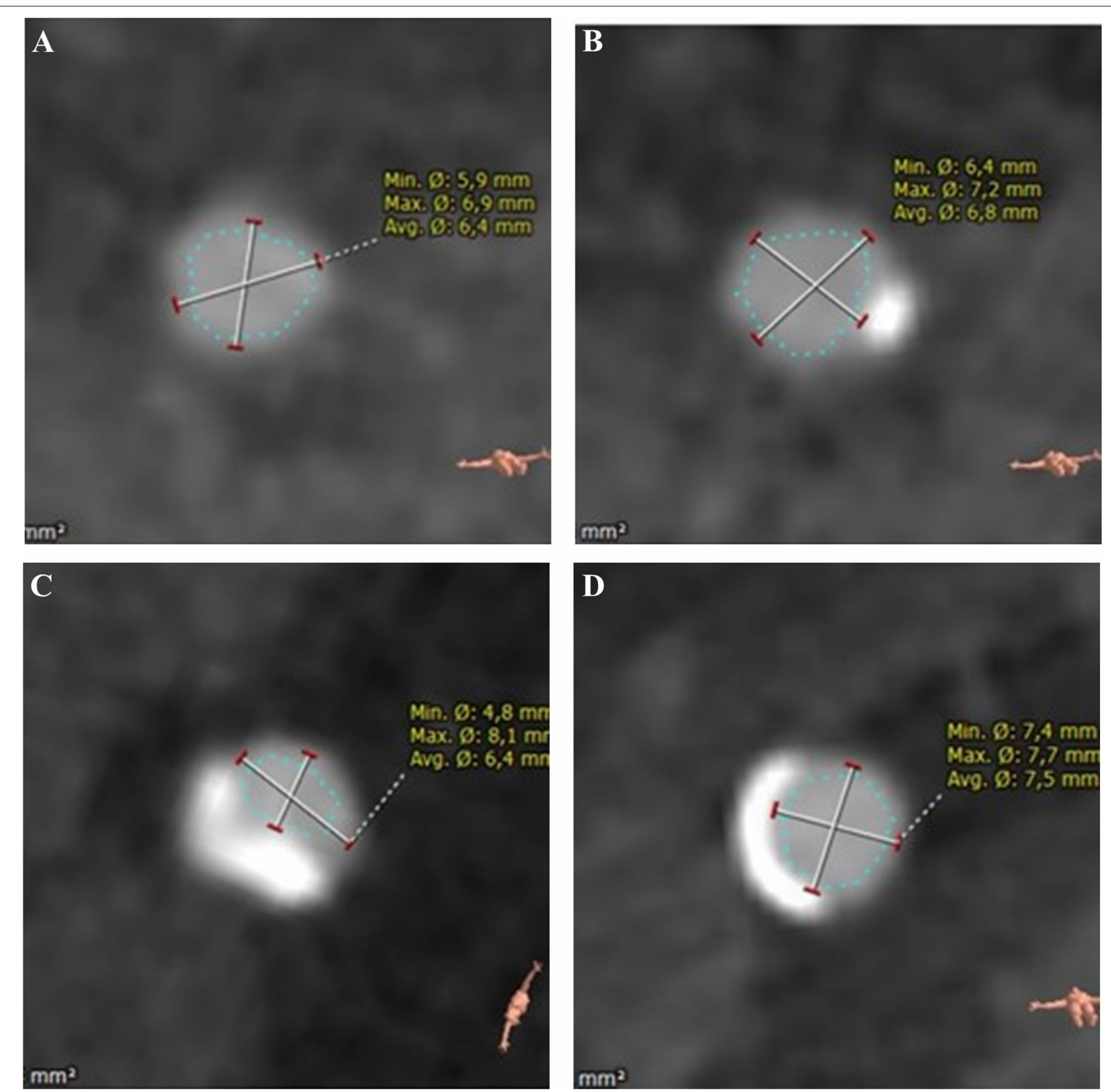

FIGURE 1 | Quantification of calcification of common femoral artery (derived from CT): none (A) mild (B) severe (C,D).

N.V., Leuven, Belgium), or in case of use of a covered stent at the access site requiring a sheath size of 8-10 French by one ProGlide. Postprocedural, there was a clinical evaluation of the access and non-access site. Any hematoma of the access site was documented. Patients with periprocedural findings other than none or mild leak or patients with suspicious findings in the postprocedural clinical examination had Doppler-/Duplex ultrasound evaluation of access site and non-access site. If this showed pseudoaneurysm, either manual compression therapy was used or an injection of thrombin.
The last angiogram of the access site was evaluated retrospectively concerning any sign of leaks, dissection/endovascular flap, stenosis (lumen reduction of $\geq 50 \%$ ), and total occlusion of the CFA (Figure 2 ).

The baseline characteristics of the patients, including medication and relevant medical history, were documented, as well as the clinically relevant periprocedural data. We assessed the sheath to femoral artery ratio (SFAR) by dividing the outer diameter of the sheath $(6.667 \mathrm{~mm}$ for the Medtronic system and $7.9 \mathrm{~mm}$ for the Boston Lotus system) by the diameter of the CFA 

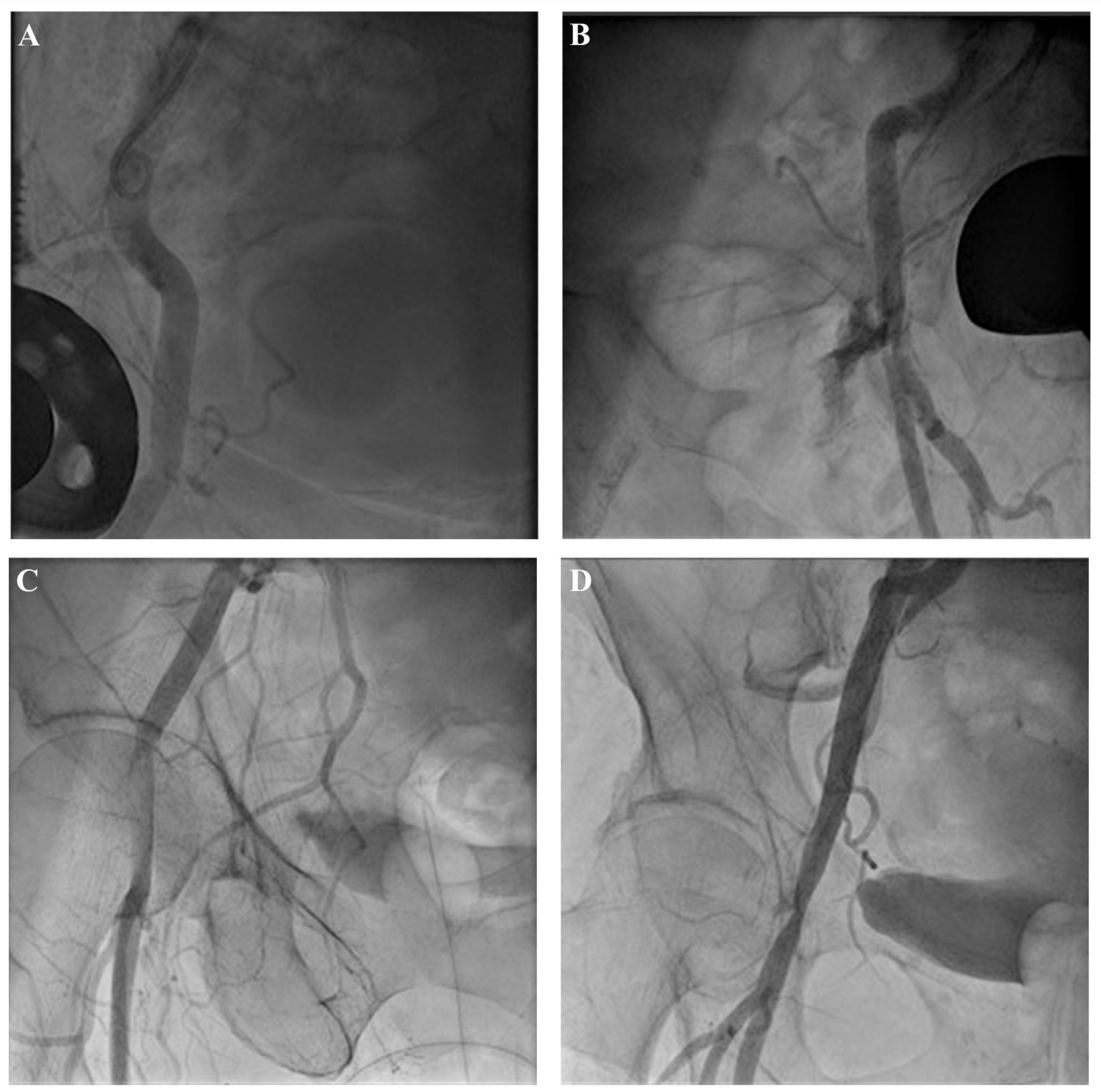

FIGURE 2 | Evaluation of angiogram after use of closure device: minor leak (A), severe leak (B), dissection (C), and stenosis with minor leaks (D).

at the expected puncture position of the access site, evaluating SFAR using the minimal diameter as well as the mean diameter.

The primary endpoint of this analysis was the predefined VARC-2 endpoints vascular access site and access-related complications with the focus on the access site itself. Major VCs at the access site were defined as vascular injuries such as perforation, bleeding, hematoma, dissection, stenosis, or pseudoaneurysm, leading to death, life-threatening or major bleeding. Minor VCs were assessed at the access and non-access site and defined as vascular injury as mentioned for the major VC however not leading to death, life-threatening, or major bleeding. Due to the small number of major VCs, patients with minor or major VC were combined and compared with patients without VC.

Furthermore, clinically relevant VARC-2 defined endpoints pacemaker, bleeding, peri-interventional stroke/transitory ischemic attack (TIA), and device success were assessed.

\section{Statistical Analysis}

We performed a sample size calculation based on assumed incidences for the small and the large sheath. Chen et al. had evaluated predictors for suture device failure and vascular 


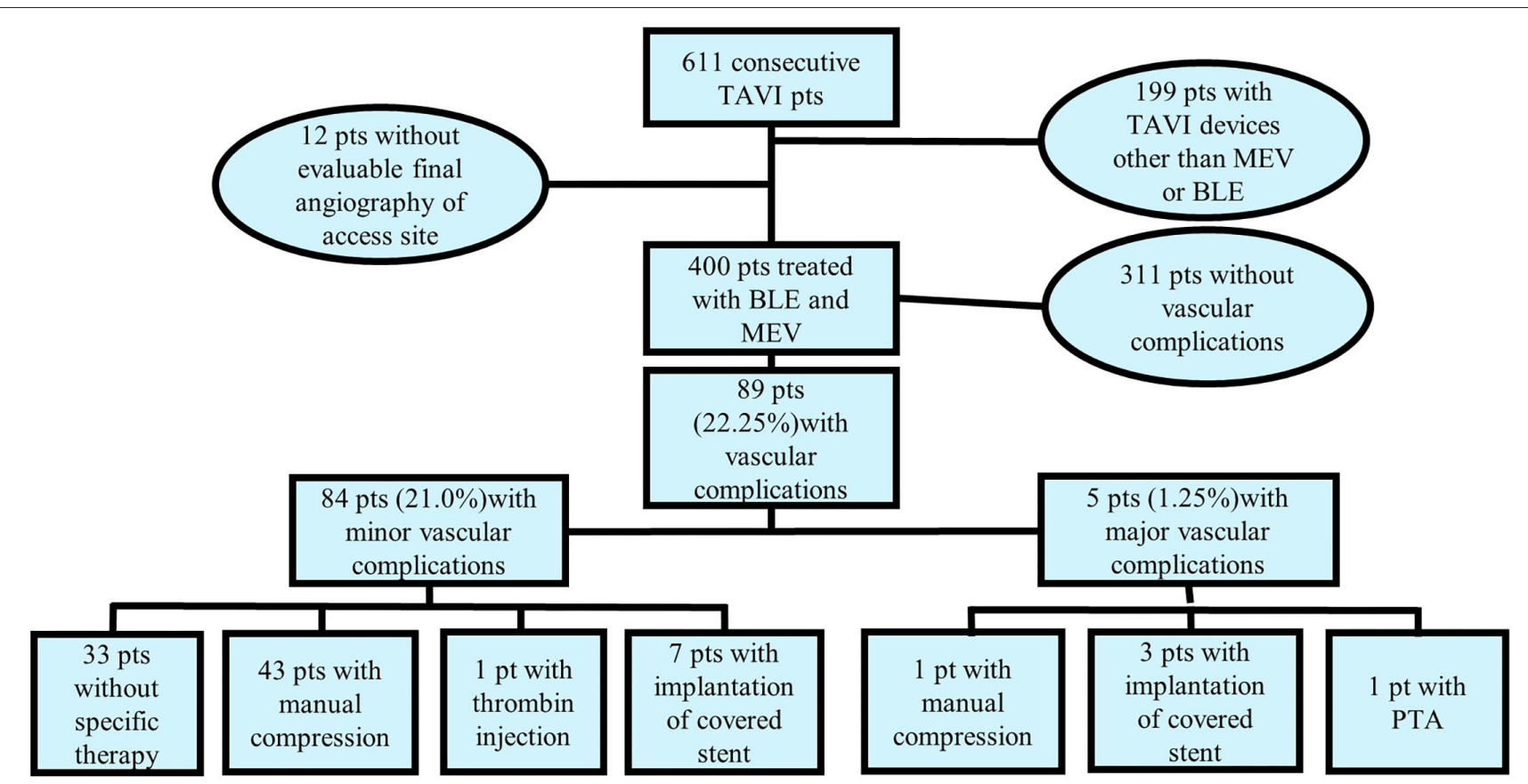

FIGURE 3 | Treatments for patients with minor and major access site vascular complications.

complications in a patient cohort of 458 patients with interventions with sheath sizes 16 to 26 French (18). The study showed a significantly higher incidence of suture device failure and following vascular complications with the use of $>21$ French sheath size than the $\leq 21 \mathrm{~F}$ sheath size with $17.2 \%$ vs. $4.9 \%, p$ $<0.001$; using a receiver operating characteristic (ROC) curve sheath, size $\geq 19$ French was found to be significantly associated with suture device failure. Barbanti et al. had experienced comparable results in their study on 375 patients ( $\geq 19$ French rates of major VC 17.5 vs. $5.9 \%, p<0.001)(20)$. Based on these results with expected event rates of $15 \%$ for the 20 French and 5\% for the 16 French access and a power of $80 \%$ and an $\alpha$-level of 0.05 group sizes of 141 patients each were found to be adequate. However, to achieve a sufficient safety margin we aimed for 200 consecutive patients with each valve type/sheath size. Statistical analysis was performed with the MedCalc software (MedCalc Version 19.6, MedCalc Software Ltd, Ostend; Belgium). Continuous variables are expressed as mean \pm one SD and were compared with the $t$-test. Categorical variables are presented as counts and percentages and differences between proportions were calculated by using the $\chi^{2}$ test. A logistic univariate and multivariate regression analysis were performed to identify predictors for VC after TAVI, results presented as odds ratio with $95 \%$ confidence interval (CI). A value of $p<0.05$ was considered statistically significant.

\section{RESULTS}

In all 400 patients, an aortic valve prosthesis was successfully implanted, in 399 patients one valve, in one patient there was the implantation of a second valve due to an embolized MEV, without further complications. There was no conversion to surgery and there was no periprocedural death. The rate of major $\mathrm{VC}$ was low with only $1.25 \%$ ( 5 patients). Two of these patients showed severe leak and three showed severe leak and dissection of the CFA after use of the vascular closure devices. Three patients were treated with the implantation of a covered stent, one patient had percutaneous transluminal angioplasty (PTA) only of the CFA and one patient was treated with manual compression only (Figure 3). All of these patients met the criteria of major bleeding complications according to the VARC-2-criteria, even though only three of them showed hematoma of the access site afterward. Patients with major VC had a significantly larger drop in hemoglobin after the TAVI procedure $(3.78 \pm 1.93$ vs. $1.34 \pm$ $1.15 \mathrm{~g} / \mathrm{dl}, p=0.048)$, and received significantly more units of blood ( $0.75 \pm 0.96$ units of blood vs. $0.05 \pm 0.34, p<0.001)$. Minor vascular complications were more frequent with $21 \%$ (84 patients). Most of these patients had access site hematoma (66 patients), four patients developed pseudoaneurysm, and in 12 patients there was stenosis of at least $50 \%$ of the CFA by duplex ultrasound without need for further treatment. There were no major VC at the non-access site, only minor VC (69 patients, $17.25 \%$ ), the majority having a hematoma (57 patients), nine developing pseudoaneurysms, which was treated with thrombin injection or manual compression (6 patients and 3 patients, respectively), and 3 patients with stenosis of at least $50 \%$ of the CFA by duplex ultrasound without need for further treatment.

\section{Baseline Characteristics}

The baseline characteristics of the patients with and without VC are displayed in Table 1. There was no statistically 
TABLE 1 | Baseline clinical characteristics.

\begin{tabular}{|c|c|c|c|}
\hline & $\begin{array}{l}\text { No VC } n= \\
\quad 311\end{array}$ & vC $n=89$ & $P$-Value \\
\hline Age, years & $80.5 \pm 6.3$ & $79.6 \pm 6.3$ & 0.24 \\
\hline Female & $159(51.1 \%)$ & $50(56.2 \%)$ & 0.40 \\
\hline $\mathrm{BMI}\left(\mathrm{kg} / \mathrm{m}^{2}\right)$ & $27.4 \pm 5.1$ & $27.5 \pm 4.5$ & 0.86 \\
\hline Diabetes mellitus & 79 (25.4\%) & 28 (31.5\%) & 0.26 \\
\hline $\begin{array}{l}\text { Chronic renal failure } \\
\text { with dialysis }\end{array}$ & $5(1.6 \%)$ & $2(2.2 \%)$ & 0.70 \\
\hline Coronary artery disease & $164(52.9 \%)$ & $54(60.7 \%)$ & 0.20 \\
\hline $\begin{array}{l}\text { History of myocardial } \\
\text { infarction }\end{array}$ & $28(9.0 \%)$ & $10(11.2 \%)$ & 0.71 \\
\hline $\begin{array}{l}\text { History of cardiac } \\
\text { surgery }\end{array}$ & $24(7.7 \%)$ & $10(11.2 \%)$ & 0.30 \\
\hline Known PAD & 28 (9.0\%) & $8(9.0 \%)$ & 1.00 \\
\hline $\begin{array}{l}\text { History of stroke or } \\
\text { intracerebral bleeding }\end{array}$ & $46(14.8 \%)$ & $11(12.4 \%)$ & 0.56 \\
\hline Pulmonary disease & $126(40.5 \%)$ & $43(48.3 \%)$ & 0.20 \\
\hline NYHA class III/IV & 207 (66.6\%) & 65 (73\%) & 0.25 \\
\hline Ejection fraction (\%) & $51.1 \pm 11.0$ & $52.6 \pm 9.0$ & 0.26 \\
\hline STS PROM & $3.48 \pm 2.37$ & $3.52 \pm 2.58$ & 0.89 \\
\hline \multicolumn{4}{|l|}{ Medication at baseline } \\
\hline ASA only & 135 (43.4\%) & $42(47.2 \%)$ & 0.53 \\
\hline DAPT & $43(13.8 \%)$ & $19(21.3 \%)$ & 0.08 \\
\hline Oral anticoagulation & 109 (35.5\%) & 22 (24.7\%) & 0.06 \\
\hline
\end{tabular}

Values are mean $\pm S D$ or $n(\%)$.

BMI, Body mass index; PAD, peripheral artery disease; NYHA, New York Heart Association; STS-PROM, Society of Thoracic Surgeons predicted risk of mortality; ASA, acetylsalicylic acid; DAPT, dual antiplatelet therapy.

significant difference concerning their medical history or clinical presentation. There was especially no difference in gender (female $51.1 \%$ in no-VC vs. $56.2 \%, p=0.1$ ), in body mass index $\left(27.4 \pm 5.1 \mathrm{~kg} / \mathrm{m}^{2}\right.$ in no-VC group vs. $27.5 \pm 4.5 \mathrm{~kg} / \mathrm{m}^{2}$ in $\mathrm{VC}$ group, $p=0.86$ ) or known history of PAD (9\% in both groups). There was a trend of more patients being treated with periinterventional dual antiplatelet therapy in the cohort of patients with VC (21.3 vs. $13.8 \%, p=0.08)$.

\section{CT Evaluation of Access Site Vessel}

The evaluation of the preprocedural CT data revealed no significant difference in the diameter of the access vessel in patients treated with the $\operatorname{BLE}(7.5 \pm 1.23 \mathrm{~mm})$ or the $\mathrm{MEV}$ $(7.36 \pm 1.51 \mathrm{~mm}, p=0.30)$. Seventeen patients $(8.5 \%)$ treated with $\mathrm{MEV}$ had a vessel diameter smaller than the formally recommended $5.5 \mathrm{~mm}, 36(18 \%)$ treated with BLE a had vessel diameter smaller than the recommended $6.5 \mathrm{~mm}$. Patients with and without VC exhibited no relevant differences between the groups concerning access vessel characteristics as well (Table 2). Thirty-six percent of the patients without VC had calcification of the access vessel and $31.5 \%$ for the patients with $\mathrm{VC}(p=0.43)$.

\section{Procedural Data}

The most frequently implanted size of BLE was $25 \mathrm{~mm}$ (43\%), followed by $27 \mathrm{~mm}$ (38\%), and $23 \mathrm{~mm}$ (19\% of BLE). For the
TABLE 2 | Characteristics of access site common femoral artery.

\begin{tabular}{lccc}
\hline & No vC $\boldsymbol{n}=\mathbf{3 1 1}$ & VC $\boldsymbol{n}=\mathbf{8 9}$ & $\boldsymbol{P}$-Value \\
\hline Minimal diameter of CFA, mm & $6.73 \pm 1.21$ & $6.58 \pm 1.69$ & 0.36 \\
Maximum diameter or CFA, mm & $8.17 \pm 1.47$ & $8.08 \pm 1.74$ & 0.65 \\
Mean diameter of CFA, mm & $7.45 \pm 1.29$ & $7.33 \pm 1.68$ & 0.49 \\
SFAR (mean diameter) & $1.01 \pm 0.20$ & $1.02 \pm 0.29$ & 0.74 \\
SFAR (minimal diameter) & $1.13 \pm 0.24$ & $1.18 \pm 0.55$ & 0.18 \\
SFAR $\geq 1.05$ (mean diameter) & $120(38.7 \%)$ & $33(37.5 \%)$ & 0.84 \\
SFAR $\geq 1.05$ (minimal diameter) & $200(64.5 \%)$ & $50(56.8 \%)$ & 0.19 \\
Calcification of CFA & & & \\
None & $199(64.0 \%)$ & $61(68.5 \%)$ & 0.64 \\
Moderate & $78(25.1 \%)$ & $18(20.2 \%)$ & \\
Severe & $34(10.9 \%)$ & $10(11.2 \%)$ & \\
\hline
\end{tabular}

Values are mean $\pm S D$ or $n(\%)$.

CFA, common femoral artery; SFAR, sheath to femoral artery ratio.

TABLE 3 | Procedural data.

\begin{tabular}{lccc}
\hline & No VC $\boldsymbol{n}=\mathbf{3 1 1}$ & VC $\boldsymbol{n}=\mathbf{8 9}$ & $\boldsymbol{P}$-Value \\
\hline Valve type & & & \\
MVE & $143(46.0 \%)$ & $57(64.0 \%)$ & 0.003 \\
BLE & $168(54.0 \%)$ & $32(36.0 \%)$ & \\
$\begin{array}{l}\text { Outer diameter of } \\
\text { sheath/delivery }\end{array}$ & $7.33 \pm 0.62$ & $7.11 \pm 0.60$ & 0.003 \\
catheter, mm & & & \\
Angiography of access site & & & \\
Leak & $159(51.1 \%)$ & $53(59.6 \%)$ & 0.16 \\
$\begin{array}{l}\text { Dissection/flap } \\
\text { Stenosis/occlusion }\end{array}$ & $75(15.4 \%)$ & $15(17.2 \%)$ & 0.26 \\
& $7(2.3 \%)$ & $4(4.5 \%)$ & 0.26 \\
\hline
\end{tabular}

Values are mean $\pm S D$ or $n(\%)$.

MVE, Medtronic evolut; BLE, Boston lotus edge; SFAR, Sheath to common femoral artery ratio.

$\mathrm{MEV}$, the most frequently implanted size was $29 \mathrm{~mm}(35.5 \%)$ followed by $26 \mathrm{~mm}$ (29\%), $34 \mathrm{~mm}$ (28\%), and $23 \mathrm{~mm}$ (7.5\%). The procedural data with respect to VC are shown in Table 3. Patients receiving MEV had minor VC more often ( 64 vs. $36 \% p=0.003)$, whereas major VC differed only numerically without statistical significance ( 2 vs. $0.5 \%, p=0.18$ ). In relation to the difference in valve type distribution concerning VC outer sheath/delivery, the catheter diameter was smaller in patients with VC. SFAR however was not associated with VC in the study cohort $(p=0.74$ for SFAR derived from mean CFA diameter; $p=0.18$ for SFAR derived from minimal CFA diameter.) The results of the final angiography of the access site after use of the closure device (and before possible intervention are displayed in Figure 4. A leak of any sort was quite frequent with the $41.5 \%$, however, the results of the angiography were not predictive for VC.

\section{Postprocedural Data}

Bleeding complications occurred in 50 patients (12.5\%), most of them being minor bleedings (33 patients, $8.25 \%$ ) due to access site or non-access site hematoma. Twelve patients developed major bleedings (3\%) and five patients had life-threatening 


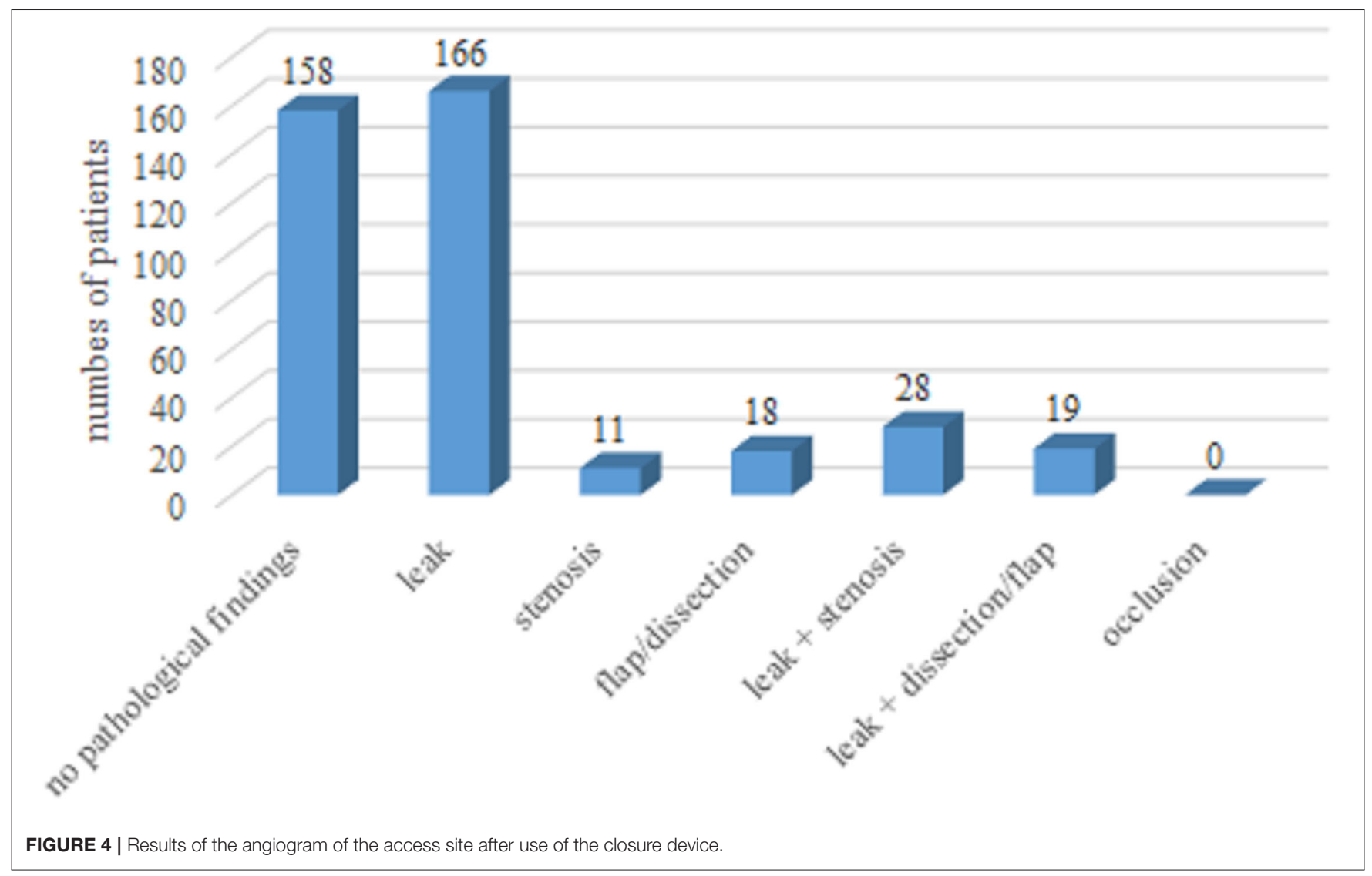

TABLE 4 | Procedural outcome.

\begin{tabular}{lccc}
\hline & No VC $\boldsymbol{n}=\mathbf{3 1 1}$ & VC $\boldsymbol{n}=\mathbf{8 9}$ & $\boldsymbol{P}$-Value \\
\hline Device success & $305(98.1 \%)$ & $87(97.8 \%)$ & 0.85 \\
Periprocedural stroke/TIA & $10(3.3)$ & $4(4.5 \%)$ & 0.56 \\
Pacemaker implantation & $67(21.5 \%)$ & $10(11.2 \%)$ & 0.03 \\
Bleeding complications & & & \\
Minor bleeding & $17(5.5 \%)$ & $16(18.0 \%)$ & 0.0002 \\
Major bleeding & $6(1.9 \%)$ & $6(6.7 \%)$ & 0.02 \\
Life threatening bleeding & $4(1.2 \%)$ & $1(1.1 \%)$ & 0.90 \\
\hline
\end{tabular}

Values are mean $\pm S D$ or $n(\%)$.

TIA, transitory ischemic attack.

bleedings, none of these access sites or non-access sites related there being two periprocedural intracerebral bleedings and three hemorrhagic pericardial effusions after pacemaker implantation after TAVI. Due to the definition of vascular complications minor and major bleeding complications occurred significantly more often in the group of patients with VC. The postprocedural pacemaker implantation rate was $19.25 \%$ for the overall population, significantly more frequent after treatment with the BLE (25\%) compared with the MEV (13.5\%, $p=0.004)$. The rate of device success was high at $98 \%$. Data on postprocedural outcomes concerning VC are presented in Table 4.

\section{Logistic Regression Analysis}

The parameters that were distributed significantly different between groups or had been identified to be predictive of VC in previous studies such as age, gender, body mass index (BMI), PAD, large sheath size, SFAR, calcification, and DAPT were entered into a univariate and multivariate logistic regression analysis (Table 5). Peri-interventional treatment with DAPT showed to be an independent predictor for VC (OR 2.11, 95\% CI $1.10-4.06, p=0.025)$. Large sheath size was not independently associated with a higher rate of vascular complications.

\section{DISCUSSION}

With the use of the newest generation TAVI devices, MEV and BLE, we report a low rate of access site-related major VC with only $1.25 \%$. Facing the low event rate, specific predictors of major VC could not be determined in this study. For the whole entity of VC combining major and minor VC peri-interventional treatment with DAPT showed to be an independent predictor for the occurrence of VC. We could not verify any of the other previous studies that identified predictors of VC.

The data on VC with the newer generation TAVI devices are still limited, most of the studies have evaluated the balloonexpandable valve with its expandable sheath. There are only a few studies on the MEV Pro valve: Major VC ranging from 0 to $10 \%$ in the first two studies, with only 60 and 74 patients, however, 
TABLE 5 | Predictors of vascular complications.

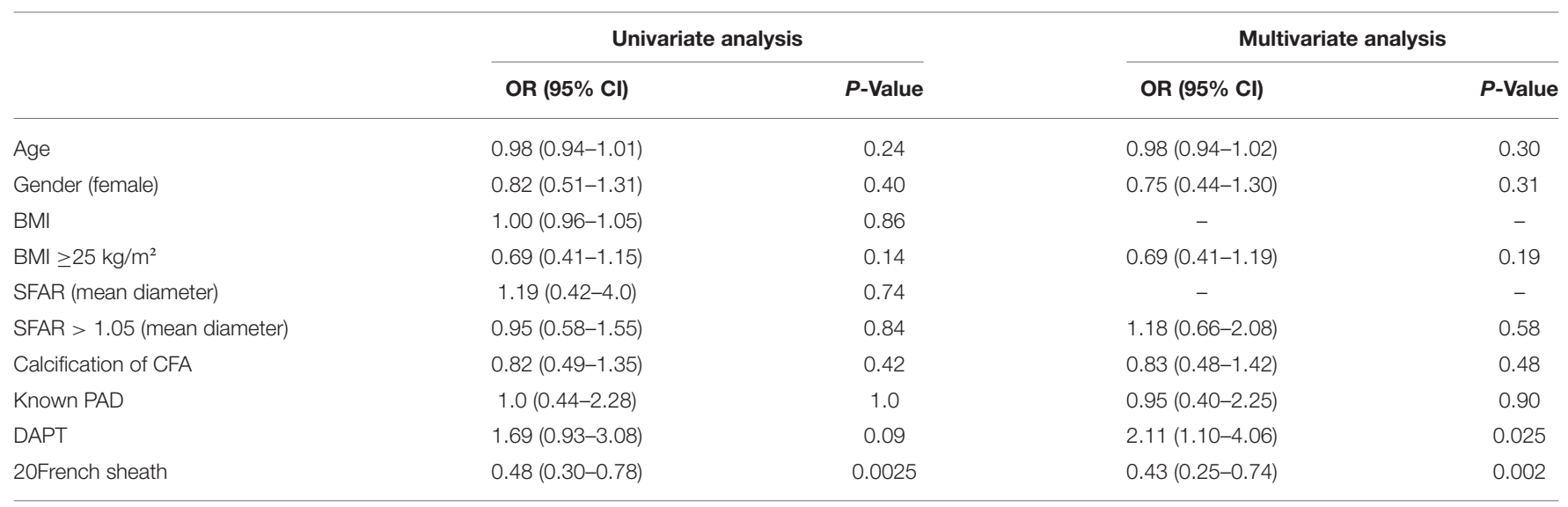

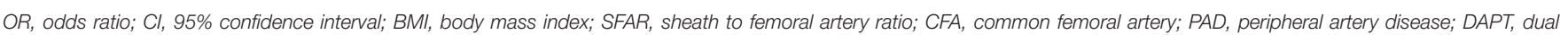
antiplatelet therapy.

the largest published study cohort with 629 patients treated with the MEV Pro (and thus the EnveoPro delivery system) had a rate of only $3.3 \%$ major VC $(15,16,23)$. Concerning the BLE valve there are no comparable data published yet, however, rates of major VC with its predecessor, the Boston Lotus valve were slightly higher ranging from 2.9 to $7.5 \%(23,24)$. Our even lower rate of major VC in this study could be explained by the fact that the evaluated study cohort recently treated between 2019 and 2020, consisted of a lower risk cohort with a Society of Thoracic Surgeons-predicted risk of mortality (STS-PROM)score $<4 \%$, furthermore, the study center being a high volume TAVI center with more than 400 procedures per year with a consistent team of experienced operators. In an analysis from the France TAVI Registry, Beurtheret et al. found a decline in major vascular complications between the period 2013-2015 and $2016 / 2017$ of 1.44 to $1.02 \%(p=0.005)$ and referring this to the increase in performed TAVI procedures from the first to the second period (25).

Despite the low rate of major VC, access site-related minor VC was still frequent with $21 \%$., because of a high rate of minor VC, mainly due to local hematoma occurring in the postprocedural period. The incidence of minor $\mathrm{VC}$ varies widely in the literature from 2 to almost $30 \%$ if mentioned at all in publications. For studies with retrospective analysis, this could be explained by center-specific differences in thoroughness on documentation of clinically non-significant access site hematoma, which already accounts as minor VC according to the VARC-2 criteria. In contrast to the negative impact of major $\mathrm{VC}$ on mortality, there is no evidence, that minor VC is associated with increased mortality or increased length of hospital stay (8).

\section{Vessel Size/Sheath Size}

In TAVI studies or studies about large endovascular access for percutaneous procedures, a variety of parameters have been indicated to influence the occurrence of VC. The most obvious seems to be the size of the access vessel, respectively, sheath size, and sheath to access site vessel ratio. Especially the ratio of the sheath to the (ilio)-femoral vessel at the access site has been described as a predictor for major VC. In the study by Hayashida et al., an SFAR threshold of 1.05 was found to be predictive for major VC, in the study by Toggweiler et al., a threshold of $1.0(7,8)$. For the newer generation, TAVI devices S(I)FAR has been confirmed as a predictor for major VC by van Kesteren et al. for the balloon-expandable Edwards Sapien 3 valve and its expandable sheath [unadjusted OR 7.51 (1.61-34.95), $p$ $=0.01)$, though the area under the curve was much lower in comparison to studies describing S(I)FAR with older generation TAVI devices, indicating poorer accuracy (13). Potluri et al. also experienced SFAR as independently associated with VC, again, a study with the Edwards Sapien 3 as the most commonly used device with its expandable sheath (19). To our knowledge, there are no published data on predictors of VC concerning the ratio of the sheath to the access site vessel regarding new generation nonexpandable sheaths. In contrast to previous studies, especially the large meta-analysis by Ueshima et al. we evaluated a recently treated low-risk patient cohort with low STS-PROM score, low rate of PAD, and use of new generation non-expandable sheaths, which might have led to the fact, that we did not experience higher rates of VC in patients with the use of the larger sheath or a higher SFAR (17). Furthermore, we only used the ProGlide vascular closure device, whereas, in older studies, other vascular closure devices as the Prostar (Abbott Vascular, Santa Clara, CA, USA) had still been in use. Mehilli et al. (12) and Seeger et al. (10) could both show in their studies that there were significantly fewer VC complications and bleeding complications with the use of ProGlide in comparison to the Prostar device.

A certain bias of not treating patients with more complex vascular access routes and smaller diameters with the larger sheath TAVI device cannot be completely excluded since the choice of the used TAVI device was not defined by a prospective randomized study design. However, there was no significant difference in vessel diameters concerning the CFA between the two valve types. Interestingly, the logistic regression analysis showed the larger sheath size to be associated with less VC, however, that statement has its limitations since this was contrary to the initial approach on this study and its power 
calculation. A further explanation could lay within the necessity of removal of the sheath for the smaller sheath size (MEV) before insertion of the delivery system with subsequent intermittent manual compression.

Throughout the published studies on VC, the measurement of SFAR does not seem to be consistently defined. In some studies, the reference for the sheath diameter is the inner diameter, in others the outer diameter. This is correlated to the mean or the minimal diameter of the access vessel or even concerning the smallest diameter of the complete iliofemoral vessel length.

\section{Calcification}

There is no unified definition/quantification of access vessel calcification yet. We tried to construct a simple semi-quantitative definition based on the CT data. However, we failed to identify severe calcification or calcification in general of the CFA as a predictor for major or minor VC. Some older reports have demonstrated that iliofemoral calcification, assessed semiquantitatively and slightly different in every report, is predictive of major VC (7). However, newer reports with more detailed CT evaluation of calcification, as Fonseca et al. who defined specific calcium thresholds, could not confirm this (14).

\section{Periprocedural Medication}

Dual antiplatelet therapy (DAPT) is known to increase bleeding risk in general and in TAVI, if that translates to a higher risk of $\mathrm{VC}$ is still not evident. Hioki et al. evaluated 540 TAVI patients and found DAPT to be a significant predictor for bleedings, however, this did not translate into a higher rate of $\mathrm{VC}$, with only approximately one-third of bleedings being associated with the access site (26). In our study cohort, peri-interventional treatment with DAPT was independently associated with a higher rate of VC.

\section{Peripheral Artery Disease}

The presence of PAD seems to be an obvious parameter for the occurrence of $\mathrm{VC}$, though existing data are not consistent. In previous studies, that have identified PAD as a predictor for VC, the rate of VC usually varied around $20 \%(13,17)$. In our study, we could not confirm the influence of that parameter, however, we only saw a low rate of reported PAD with $9 \%$ in our study cohort, as well as Fonseca et al. who described a rate of $11.4 \%$ and could not identify PAD as a predictor for VC as well (14). The low reported incidence of reported PAD in our cohort may be attributed to the cohort being a low-risk cohort with a mean STS-PROM of $<4 \%$.

\section{LIMITATIONS}

Our study has several limitations. First, it is a retrospective singlecenter analysis. The lack of randomization may influence the selection of patients and outcomes. As for the documentation of minor vascular complications, not every patient had a duplex ultrasound of the access site post procedurally, only those with already clinically suspected pathological findings. Therefore, it is possible to have overlooked some minor VC complications.
The study was powered for the parameter sheath size and a difference of at least $10 \%$. Therefore, we cannot derive definitive conclusions on other parameters, and cannot exclude, that smaller differences in predicting values of sheath diameter are present and are not detected for power limitations.

\section{CONCLUSION}

With the newest generation of TAVI devices, major VC seems to have reached the bottom line. The incidence of $\mathrm{VC}$ was low and most of the previously detected potential risk factors showed no relevant influence on the occurrence of VC in general in our study population of 400 patients, neither on major VC and thus did not offer potential angles for optimization. Only peri-interventional treatment with DAPT was associated with the occurrence of VC. Most likely, these low major VC rates are nowadays achieved by device improvements concerning sheaths and delivery systems, profound knowledge of performing percutaneous vascular access with large diameters, and wellestablished endovascular treatment options in case of need.

\section{DATA AVAILABILITY STATEMENT}

The raw data supporting the conclusions of this article will be made available by the authors, without undue reservation.

\section{ETHICS STATEMENT}

The studies involving human participants were reviewed and approved by Ethics Committee of the University of Ulm, Ulm Germany. The patients/participants provided their written informed consent to participate in this study.

\section{AUTHOR CONTRIBUTIONS}

BG: conceptualization, investigation, validation, methodology, formal analysis, and writing-original draft. $\mathrm{CR}$ and JM: investigation and data curation. WR: funding acquisition conceptualization, investigation, supervision, writing-review, and editing. DB: conceptualization, investigation, methodology, formal analysis, supervision, writing-review, and editing. All persons who meet the authorship criteria are listed as authors, certify that they have participated sufficiently in the work to take public responsibility for the content, including participation in the concept, design, analysis, writing, or revision of the manuscript, and provided critical feedback to the manuscript and approved the final version of the manuscript.

\section{FUNDING}

For the conduction of the study, the Department of Internal Medicine II, University of Ulm, received restricted funds from Medtronic (Medtronic Minneapolis, Minnesota, USA). The funder was not involved in the study design, collection, analysis, interpretation of data, the writing of this article, or the decision to submit it for publication. 


\section{REFERENCES}

1. Cribier A, Eltchaninoff H, Bash A, Borenstein N, Tron C, Bauer F, et al. Percutaneous transcatheter implantation of an aortic valve prosthesis for calcific aortic stenosis: first human case description. Circulation. (2002) 106:3006-8. doi: 10.1161/01.CIR.0000047200.36165.B8

2. Makkar RR, Fontana GP, Jilaihawi H, Kapadia S, Pichard AD, Douglas PS, et al. Transcatheter aortic-valve replacement for inoperable severe aortic stenosis. $N$ Engl J Med. (2012) 366:1696-704. doi: 10.1056/NEJMoa1202277

3. Reardon MJ, Van Mieghem NM, Popma JJ, Kleiman NS, Sondergaard L, Mumtaz M, et al. Surgical or transcatheter aortic-valve replacement in intermediate-risk patients. $N$ Engl J Med. (2017) 376:1321-31. doi: 10.1056/NEJMoa1700456

4. Anantha-Narayanan M, Kandasamy VV, Reddy YN, Megaly M, Baskaran J, Pershad A, et al. Low-Risk transcatheter versus surgical aortic valve replacement - an updated meta-analysis of randomized controlled trials. Cardiovasc Revasc Med. (2020) 21:441-52. doi: 10.1016/j.carrev.2019. 08.003

5. Pascual I, Carro A, Avanzas P, Hernandez-Vaquero D, Diaz R, Rozado J, et al. Vascular approaches for transcatheter aortic valve implantation. J Thorac Dis. (2017) 9 (Suppl. 6):S478-87. doi: 10.21037/jtd.2017.05.73

6. Genereux P, Head SJ, Van Mieghem NM, Kodali S, Kirtane AJ, Xu K, et al. Clinical outcomes after transcatheter aortic valve replacement using valve academic research consortium definitions: a weighted meta-analysis of 3,519 patients from 16 studies. J Am Coll Cardiol. (2012) 59:2317-26. doi: $10.1016 /$ j.jacc.2012.02.022

7. Toggweiler S, Gurvitch R, Leipsic J, Wood DA, Willson AB, Binder RK, et al. Percutaneous aortic valve replacement: vascular outcomes with a fully percutaneous procedure. J Am Coll Cardiol. (2012) 59:113-8. doi: 10.1016/j.jacc.2011.08.069

8. Hayashida K, Lefevre T, Chevalier B, Hovasse T, Romano M, Garot P, et al. Transfemoral aortic valve implantation new criteria to predict vascular complications. JACC Cardiovasc Interv. (2011) 4:851-8. doi: 10.1016/j.jcin.2011.03.019

9. Gurvitch R, Toggweiler S, Willson AB, Wijesinghe N, Cheung A, Wood DA, et al. Outcomes and complications of transcatheter aortic valve replacement using a balloon expandable valve according to the valve academic research consortium (VARC) guidelines. EuroIntervention. (2011) 7:41-8. doi: 10.4244/EIJV7I1A10

10. Seeger J, Gonska B, Rodewald C, Rottbauer W, Wohrle J. Impact of suture mediated femoral access site closure with the prostar XL compared to the ProGlide system on outcome in transfemoral aortic valve implantation. Int J Cardiol. (2016) 223:564-7. doi: 10.1016/j.ijcard.2016.08.193

11. Ducrocq G, Francis F, Serfaty JM, Himbert D, Maury JM, Pasi N, et al. Vascular complications of transfemoral aortic valve implantation with the edwards SAPIEN prosthesis: incidence and impact on outcome. EuroIntervention. (2010) 5:666-72. doi: 10.4244/EIJV5I6A110

12. Mehilli J, Jochheim D, Abdel-Wahab M, Rizas KD, Theiss H, Spenkuch $\mathrm{N}$, et al. One-year outcomes with two suture-mediated closure devices to achieve access-site haemostasis following transfemoral transcatheter aortic valve implantation. EuroIntervention. (2016) 12:1298-304. doi: 10.4244/EIJV12I10A213

13. van Kesteren F, van Mourik MS, Vendrik J, Wiegerinck EMA, Henriques JPS, Koch KT, et al. Incidence, predictors, and impact of vascular complications after transfemoral transcatheter aortic valve implantation with the SAPIEN 3 prosthesis. Am J Cardiol. (2018) 121:1231-8. doi: 10.1016/j.amjcard.2018.01.050

14. Fonseca P, Almeida J, Bettencourt N, Ferreira N, Carvalho M, Ferreira W, et al. Incidence and predictors of vascular access site complications following transfemoral transcatheter aortic valve implantation. Rev Port Cardiol. (2017) 36:747-53. doi: 10.1016/j.repc.2015.11.009

15. Hellhammer K, Piayda K, Afzal S, Kleinebrecht L, Makosch M, Hennig I, et al. The latest evolution of the medtronic CoreValve system in the era of transcatheter aortic valve replacement: matched comparison of the evolut PRO and evolut R. JACC Cardiovasc Interv. (2018) 11:2314-22. doi: 10.1016/j.jcin.2018.07.023

16. Forrest JK, Mangi AA, Popma JJ, Khabbaz K, Reardon MJ, Kleiman NS, et al. Early outcomes with the evolut PRO repositionable self-expanding transcatheter aortic valve with pericardial wrap. JACC Cardiovasc Interv. (2018) 11:160-8. doi: 10.1016/j.jcin.2017.10.014

17. Ueshima D, Barioli A, Nai Fovino L, D’Amico G, Fabris T, Brener SJ, et al. The impact of pre-existing peripheral artery disease on transcatheter aortic valve implantation outcomes: a systematic review and meta-analysis. Catheter Cardiovasc Interv. (2020) 95:993-1000. doi: 10.1002/ccd.28335

18. Chen IM, Lee TH, Chen PL, Shih CC, Chang HH. Factors in ProGlide(R) vascular closure failure in sheath arteriotomies greater than 16 french. Eur J Vasc Endovasc Surg. (2019) 58:615-22. doi: 10.1016/j.ejvs.2019.03.037

19. Potluri SP, Hamandi M, Basra SS, Shinn KV, Tabachnick D, Vasudevan A, et al. Comparison of frequency of vascular complications with ultrasoundguided versus fluroscopic roadmap-guided femoral arterial access in patients who underwent transcatheter aortic valve implantation. Am J Cardiol. (2020) 132:93-9. doi: 10.1016/j.amjcard.2020.07.013

20. Barbanti M, Binder RK, Freeman M, Wood DA, Leipsic J, Cheung A, et al. Impact of low-profile sheaths on vascular complications during transfemoral transcatheter aortic valve replacement. EuroIntervention. (2013) 9:929-35. doi: 10.4244/EIJV9I8A156

21. Kappetein AP, Head SJ, Genereux P, Piazza N, van Mieghem NM, Blackstone $\mathrm{EH}$, et al. Updated standardized endpoint definitions for transcatheter aortic valve implantation: the valve academic research consortium- 2 consensus document. J Am Coll Cardiol. (2012) 60:1438-54. doi: 10.1093/ejcts/ezs533

22. Baumgartner H, Falk V, Bax JJ, De Bonis M, Hamm C, Holm PJ, et al. 2017 ESC/EACTS guidelines for the management of valvular heart disease. Eur Heart J. (2017) 38:2739-91. doi: 10.1016/j.rec.2017.12.013

23. Reardon MJ, Feldman TE, Meduri CU, Makkar RR, O’Hair D, Linke A, et al. Two-Year outcomes after transcatheter aortic valve replacement with mechanical vs self-expanding valves: the REPRISE III randomized clinical trial. JAMA Cardiol. (2019) 4:223-9. doi: 10.1001/jamacardio.2019.0091

24. Seeger J, Gonska B, Rottbauer W, Wohrle J. New generation devices for transfemoral transcatheter aortic valve replacement are superior compared with last generation devices with respect to VARC-2 outcome. Cardiovasc Interv Ther. (2018) 33:247-55. doi: 10.1007/s12928-017-0477-6

25. Beurtheret S, Karam N, Resseguier N, Houel R, Modine T, Folliguet $\mathrm{T}$, et al. Femoral versus nonfemoral peripheral access for transcatheter aortic valve replacement. J Am Coll Cardiol. (2019) 74:2728-39. doi: 10.1016/j.jacc.2019.09.054

26. Hioki H, Watanabe Y, Kozuma K, Nara Y, Kawashima H, Kataoka A, et al. Pre-procedural dual antiplatelet therapy in patients undergoing transcatheter aortic valve implantation increases risk of bleeding. Heart. (2017) 103:361-7. doi: 10.1136/heartjnl-2016-309735

Conflict of Interest: The authors declare that the research was conducted in the absence of any commercial or financial relationships that could be construed as a potential conflict of interest.

Publisher's Note: All claims expressed in this article are solely those of the authors and do not necessarily represent those of their affiliated organizations, or those of the publisher, the editors and the reviewers. Any product that may be evaluated in this article, or claim that may be made by its manufacturer, is not guaranteed or endorsed by the publisher.

Copyright (๑) 2021 Gonska, Reuter, Mörike, Rottbauer and Buckert. This is an openaccess article distributed under the terms of the Creative Commons Attribution License (CC BY). The use, distribution or reproduction in other forums is permitted, provided the original author(s) and the copyright owner(s) are credited and that the original publication in this journal is cited, in accordance with accepted academic practice. No use, distribution or reproduction is permitted which does not comply with these terms. 